\title{
Parasitos oportunistas intestinais em pacientes submetidos à hemodiálise - revisão sistemática da literatura
}

\author{
Opportunistic intestinal parasites in hemodialysis patients - a systematic literature review
}

Parásitos intestinales oportunistas en pacientes sometidos a hemodiálisis - revisión sistemática de la literatura

Solimar Almeida de Oliveira1, Sônia de Fátima Oliveira Santos², Marco Tulio Antonio García-Zapata³

\footnotetext{
${ }^{1}$ Biomédica, Mestre em Ciências da Saúde. Biomédica do Instituto de Patologia Tropical da Universidade Federal de Goiás (IPTSP/UFG). Goiânia, GO, Brasil. E-mail: solbiomed@hotmail.com.

${ }^{2}$ Bióloga, Doutora em Ciências da Saúde. Professora da Faculdade FIBRA. Anápolis, GO, Brasil. E-mail: soniaoliveirasantos@yahoo.com.br.

${ }^{3}$ Médico e Biólogo, Doutor em Infectologia e Medicina Tropical. Professor Titular do IPTSP/UFG. Goiânia, GO, Brasil. E-mail: mctulianpg@gmail.com.
}

\section{RESUMO}

Realizou-se uma revisão sistemática da literatura com o objetivo de identificar a ocorrência de parasitos entéricos oportunistas em pacientes nefropatas crônicos submetidos à hemodiálise. A revisão consistiu de busca de artigos publicados nas bases de dados MEDLINE, LILACS, SciELO, PubMed entre 1991 e 2013. Foram identificados 178 artigos, dos quais dez foram considerados relevantes para o presente trabalho. Nos trabalhos os pesquisadores demonstraram que os pacientes imunossuprimidos submetidos à hemodiálise são potencialmente infectados por agentes entéricos oportunistas. Há necessidade de maior quantidade de estudos sobre o tema uma vez que existe uma ocorrência mundial crescente das doenças renais crônicas e o potencial acometimento de doenças oportunistas nesta população podendo, inclusive, ocasionar a contaminação de ambientes hospitalares por protozoários entéricos oportunistas.

Descritores: Diálise Renal; Blastocystis hominis; Cryptosporidium; Cyclospora; Isospora.

\section{ABSTRACT}

The objective of this systematic literature review was to identify the occurrence of opportunistic enteric parasites in chronic kidney patient undergoing hemodialysis. The review consisted on searching articles published on MEDLINE, LILACS, SciELO, and PubMed databases between 1991 and 2013. A total 178 articles were identified, ten of which were considered relevant for the present study. In the referred studies, the researchers demonstrated that immunosuppressed patients undergoing hemodialysis are potentially infected by opportunistic enteric agents. Further studies are needed on this topic, as there is a growing global concern with chronic kidney diseases and the potential for these patients contracting opportunistic diseases, which, inclusively, could contaminate hospital environments with opportunistic enteric protozoa.

Descriptors: Renal Dialysis; Blastocystis hominis; Cryptosporidium; Cyclospora; Isospora.

\section{RESUMEN}

Se realizó revisión sistemática de la literatura con el objetivo de identificar la ocurrencia de parásitos entéricos oportunistas en pacientes de nefropatía crónica sometidos a hemodiálisis. La revisión consistió en la búsqueda de artículos publicados en las bases de datos MEDLINE, LILACS, SciELO y PubMed, entre 1991 y 2013 . Fueron identificados 178 artículos, de los cuales diez fueron considerados relevantes para el presente estudio. En los trabajos, los investigadores demostraron que los pacientes inmunosuprimidos sometidos a hemodiálisis son potencialmente infectados por agentes entéricos oportunistas. Hay necesidad de mayor cantidad de estudios sobre el tema, toda vez que existe presencia creciente a nivel mundial de enfermedades renales crónicas y el potencial agravamiento por enfermedades oportunistas en ésta población, pudiendo, inclusive, dar lugar a la contaminación de ambientes hospitalarios por protozoarios entéricos oportunistas.

Descriptores: Diálisis Renal; Blastocystis hominis; Cryptosporidium; Cyclospora; Isospora. 


\section{INTRODUÇÃO}

A prevalência das Doenças Renais Crônicas (DRC) tem aumentado em todo o mundo devido à maior expectativa de vida e envelhecimento da população, associada ainda, a outras doenças como diabetes mellitus (DM) e hipertensão arterial sistêmica (HAS), resultando em um problema de saúde pública quanto à incidência da doença e ao custeio de tratamento(1). A DRC é responsável por provocar perdas progressivas e irreversíveis da função renal com alterações metabólicas, hemodinâmicas, hematológicas e ósseas, levando ao estado de imunossupressão do paciente ${ }^{(2)}$.

De acordo com o National Kidney Foundation 2002 (K/DOQI 2002)(3), são 6 os estágios da DRC compreendidos em: estágio 0) função renal normal: a taxa de filtração glomerular (TFG) é maior ou igual a 90 $\mathrm{ml} / \mathrm{min} / 1,73 \mathrm{~m}^{2}$, sem lesão histológica, mas com presença de fatores de risco; estágio 1) função renal normal: TFG normal ou aumentada: maior ou igual a $90 \mathrm{ml} / \mathrm{min} / 1,73$ $\mathrm{m}^{2}$, aparecimento de lesão histológica; estágio 2) insuficiência renal funcional ou leve: TFG entre 60 e 89 $\mathrm{ml} / \mathrm{min} / 1,73 \mathrm{~m}^{2}$; estágio 3) insuficiência renal laboratorial ou moderada: TFG está entre 30 e $59 \mathrm{ml} / \mathrm{min} / 1,73 \mathrm{~m}^{2}$; estágio 4) insuficiência renal clínica ou grave: TFG entre 15 e $29 \mathrm{ml} / \mathrm{min} / 1,73 \mathrm{~m}^{2}$ e estágio 5) insuficiência renal terminal: TFG menor que $15 \mathrm{ml} / \mathrm{min} / 1,73 \mathrm{~m}^{2}$.

Na fase terminal, é necessária a submissão do paciente à terapia renal substitutiva (TRS) - hemodiálise ou transplante renal.

Associadas à DRC surgem fatores como a desnutrição, que aliada ao uso de medicamentos imunossupressores contribui para a queda da imunidade do paciente. Essa condição favorece o aparecimento de doenças causadas por agentes entéricos oportunistas, que por sua vez, também constituem um problema de saúde coletiva, sobretudo, em países em desenvolvimento(4), onde as condições qualitativas de vida da população não são proporcionais ao seu crescimento ${ }^{(5)}$.

A partir da década de 80 , com o surgimento e a disseminação da Síndrome da Imunodeficiência Adquirida (HIV/AIDS), as infecções parasitárias emergiram e se destacaram. Parasitoses que antes só eram reconhecidas na comunidade médico-veterinária(6) e raramente acometiam serem humanos, passaram, com frequência, a serem diagnosticadas em indivíduos com sistema imune deficitário, apresentando quadros agudos severos, assumindo assim, grande relevância como agentes de infecções oportunistas ${ }^{(7)}$.

Dentre as parasitoses emergentes e reemergentes, que causam doenças oportunistas, podem-se destacar os protozoários entéricos da Subclasse Coccidia, responsáveis por inúmeros casos de infecções refratárias a medicamentos, os quais podem levar o paciente imunossuprimido a apresentar quadros de diarréia, vômito, desidratação e óbito ${ }^{(7)}$.

Protozooses como as Coccidioses intestinais e as Blastocistoses são as de maior relevância, identificadas por determinarem infecções refratárias com significantes causas de morte em pacientes aidéticos com contagem de células $\mathrm{CD}_{4}$ inferior a $200 \mathrm{cel} / \mathrm{mm}^{3}$. Os Coccídeos (Phylum Aplicomplexa) são responsáveis por infectar o trato intestinal em pacientes imunossuprimidos, ocasionando diarreias prolongadas, representados principalmente por Isospora belli, Cryptosporidium sp, Sarcocystis sp e Cyclospora cayetanensis(8).

Assim, doenças causadas por protozoários entéricos oportunistas como B. hominis, Cryptosporidium sp, I. belli, C. cayetanensis adquirem crescente importância no grupo de indivíduos portadores de DRC.

Por serem os protozoários entéricos oportunistas de alta potencialidade de contaminação dos ambientes, entre eles, os hospitalares, sua detecção é necessária para impedir a transmissão da infecção associada a procedimentos de hemodiálise ${ }^{(9)}$.

Neste contexto, diante da crescente incidência da DRC e da possibilidade de sua associação às doenças parasitárias emergentes e reermegentes, faz-se necessária uma revisão sistemática da literatura com o objetivo de mapear a situação atual do conhecimento relativo à ocorrência de parasitos entéricos oportunistas em pacientes nefropatas crônicos submetidos à hemodiálise.

\section{MÉTODOS}

Trata-se de estudo de revisão sistemática da literatura(10-11) sobre a ocorrência de protozoários entéricos em hemodialisados norteado pela questão: Qual a ocorrência de Blastocystis hominis, Cryptosporidium sp, Isospora belli e Cyclospora cayetanensis em pacientes com insuficiência renal crônica submetidos à hemodiálise? 
A revisão sistemática da literatura obedeceu às seguintes fases:

\section{$1^{a}$ Fase - Elaboração do teste de relevância e seleção da base de dados}

Os pesquisadores iniciaram a investigação individualmente com a utilização de formulários padronizados denominados Testes de Relevância que obedeceram a critérios previamente estipulados de inclusão e exclusão para avaliar as questões a respeito de clareza, coerência e pertinência dos artigos com esta pesquisa, como: tipo de estudo, análise do tipo de população estudada (se de nefropatas crônicos ou não), existência ou não de dados suficientes para análise da ocorrência de protozoários entéricos na população.

A busca de artigos foi mediada por três pesquisadores, de forma individualizada, com a opção de localização das informações na Biblioteca Virtual em Saúde (BVS), pelo endereço http://www.bireme.br/ , utilizando as seguintes bases de dados: LILACS (Literatura Latino-Americana e do Caribe em Ciências da Saúde), MEDLINE (International Literature in Health Sciences) e SciELO (Scientific Eletronic Library Online), bem como pelo site da PubMed (US National Library of Medicine National Institutes of Health). Devido ao fato de a base de dados SciELO não possibilitar a pesquisa via descritores, optouse pela realização de pesquisa por palavras em todas as bases. Essas bases foram escolhidas por serem comumente consultadas como fontes de literatura qualificada dentro das Ciências da Saúde. A variação temporal foi de artigos publicados entre 1991 e 2013.

\section{$2^{\mathbf{a}}$ Fase - Seleção de descritores}

A busca por artigos científicos foi realizada no dia 26 de junho de 2013, das 14 às 18 horas.

Foram selecionados artigos nos idiomas português, inglês e espanhol, utilizando as seguintes associações de descritores: diálise renal "and" Blastocystis (DR+B); diálise renal "and" Cryptosporidium (DR+Cr); diálise renal "and" Cyclospora (DR+Cy); diálise renal "and" Isospora (DR+1); nefropatia terminal "and" Blastocystis $(\mathrm{NT}+B)$; nefropatia terminal "and" Cryptosporidium (NT+Cr); nefropatia terminal "and" Cyclospora (NT+Cy); nefropatia terminal "and" Isospora (NT+I); hemodiálise "and" Blastocystis $(\mathrm{h}+B)$; hemodiálise "and" Cryptosporidium $(\mathrm{h}+\mathrm{Cr})$; hemodiálise "and" Cyclospora $(\mathrm{h}+\mathrm{Cy})$; hemodiálise "and"
Isospora $(\mathrm{h}+\ell)$; hemodialysis "and" Blastocystis (hs+B); hemodialysis "and" Cryptosporidium (hs+Cr); hemodialysis "and" Cyclospora (hs+Cy); hemodialysis "and" Isospora (hs+1); kidney diseases "and" Blastocystis $(\mathrm{kd}+B)$, kidney diseases "and" Cryptosporidium (kd+Cr); kidney diseases "and" Cyclospora $(\mathrm{kd}+C y)$, kidney diseases "and" Isospora $(\mathrm{kd}+l)$.

\section{$3^{a}$ Fase - Seleção e análise dos artigos}

Após o levantamento dos artigos científicos nas bases de dados, estes foram submetidos à aplicação do Teste de Relevância I, por meio da leitura dos resumos. O teste foi aplicado pelos pesquisadores de forma independente e individualizada, no qual foi observado o índice de confiabilidade (IC) entre os pesquisadores segundo o cálculo: $I C=(A / A+D) \times 100$, sendo $A$, o número de acordos e $D$, o número de desacordos. Foi considerado aceitável IC $\geq \mathbf{8 0 \%}{ }^{(12)}$.

Os formulários obedeciam a critérios previamente estipulados de inclusão e exclusão contidos no Teste de Relevância I, que foi aplicado apenas aos resumos dos artigos. O Teste de Relevância II foi aplicado ao artigo na íntegra a partir dos estudos anteriormente selecionados pelo Teste de Relevância I.

A quantidade de artigos encontrados no dia 26 de junho de 2013, das 14 às 18 horas foi de 178 artigos, do quais $22(12,35 \%)$ foram localizados na base de dados LILACS, 79 (44,38\%) na base de dados MEDLINE, 08 $(4,49 \%)$ na base de dados SCIELO e $69(38,76 \%)$ na base de dados PUBMED.

A aplicação do teste de relevância I resultou na seleção de 14 artigos (7,86\%), para a análise da obra completa, excluindo 164 (92,13\%) dos artigos encontrados. $O$ índice de confiabilidade encontrado foi de $97,75 \%$.

O teste de relevância II, por sua vez, aplicado às 14 obras completas encontradas na literatura, resultou na exclusão de quatro artigos. Assim, foram finalmente selecionados 10 artigos $^{(13-22)}$ a serem analisados (Figura 1 e Quadro 1). 
Figura 1: Fluxograma das etapas para seleção dos artigos.



Legenda:

TR1: Teste de relevância I

TR2: Teste de relevância II 
Quadro 1: Síntese dos artigos selecionados destacando os agentes infecciosos pesquisados em cada estudo e sua localização geográfica, após a aplicação do Teste de Relevância I.

\begin{tabular}{|c|c|c|c|}
\hline $\begin{array}{c}\text { Autores/Base de } \\
\text { indexação do artigo }\end{array}$ & Título do artigo & Origem & $\begin{array}{l}\text { Agentes encontrados nos } \\
\text { resultados dos estudos }\end{array}$ \\
\hline \multicolumn{4}{|c|}{ LILACS } \\
\hline $\begin{array}{l}\text { Seyrafian S et al. }{ }^{(15)} \\
2006\end{array}$ & $\begin{array}{c}\text { Prevalence rate of Cryptosporidium } \\
\text { infection in hemodialysis patients in Iran. }\end{array}$ & Iran & Cryptosporidium \\
\hline $\begin{array}{l}\text { Chieffi PP et al. }{ }^{(18)} \\
1998\end{array}$ & $\begin{array}{l}\text { Infection by Cryptosporidium parvum in } \\
\text { renal patients submitted to renal } \\
\text { transplant or hemodialysis. }\end{array}$ & Brasil & Cryptosporidium \\
\hline Jabur P et al.(19) 1996 & $\begin{array}{c}\text { Criptosporidiose e outras } \\
\text { enteroparasitoses em pacientes } \\
\text { submetidos a transplante renal ou } \\
\text { hemodiálise }\end{array}$ & Brasil & Cryptosporidium \\
\hline \multicolumn{4}{|c|}{ SciELO } \\
\hline Kulik RA et al..$^{(14)} 2008$ & $\begin{array}{l}\text { Blastocystis sp and other intestinal } \\
\text { parasites in hemodialysis patients }\end{array}$ & Brasil & $\begin{array}{c}\text { Blastocystis, Endolimax nana, } \\
\text { Cryptosporidium, Entamoeba coli }\end{array}$ \\
\hline \multicolumn{4}{|c|}{ MEDLINE } \\
\hline Baqai R et al.(16) 2005 & $\begin{array}{l}\text { Detection of Cryptosporidium in } \\
\text { immunosuppressed patients }\end{array}$ & Paquistão & Cryptosporidium \\
\hline $\begin{array}{c}\text { Abaza SM et al. } \\
1995\end{array}$ & $\begin{array}{c}\text { Intestinal opportunistic parasites among } \\
\text { different groups of immunocompromised } \\
\text { hosts }\end{array}$ & Egito & Cryptosporidium \\
\hline $\begin{array}{l}\text { Baiomy AM et al. }{ }^{(13)} \\
2010\end{array}$ & $\begin{array}{l}\text { Opportunistic parasitic infections among } \\
\text { immunocompromised Egyptian patients }\end{array}$ & Egito & $\begin{array}{c}\text { Cryptosporidium, Cyclospora } \\
\text { Cayetanensis, Giardia lamblia, } \\
\text { Microsporidium }\end{array}$ \\
\hline \multicolumn{4}{|c|}{ PUBMED } \\
\hline $\begin{array}{l}\text { Turkcapar } \mathrm{N} \text { et } a^{(17)} \\
2002\end{array}$ & $\begin{array}{l}\text { Prevalence of Cryptosporidium infection in } \\
\text { hemodialysis patients }\end{array}$ & Turquia & Cryptosporidium \\
\hline Ali MS et al. ${ }^{(21)} 2000$ & $\begin{array}{l}\text { Intestinal spore-forming protozoa among } \\
\text { patients suffering from chronic renal } \\
\text { failure }\end{array}$ & Egito & $\begin{array}{l}\text { Cryptosporidium, Cyclospora } \\
\text { Cayetanensis, Entamoeba } \\
\text { histolystica, Isospora belli, } \\
\text { Microsporidium }\end{array}$ \\
\hline Gil FF et al.(22) 2013 & $\begin{array}{l}\text { Prevalence of intestinal parasitism and } \\
\text { associated symptomatology among } \\
\text { hemodialysis patients }\end{array}$ & Brasil & $\begin{array}{c}\text { Cryptosporidium, Blastocystis, } \\
\text { Endolimax nana, Entamoeba coli, E. } \\
\text { histolytica/E.coli, Strongyloides } \\
\text { stercolaris, Giardia lamblia }\end{array}$ \\
\hline
\end{tabular}

\section{RESULTADOS}

A síntese da análise dos artigos selecionados para o estudo foi apresentada no Quadro 1.

\section{DISCUSSÃO}

Existem diversos estudos sobre a incidência de parasitos entéricos oportunistas em populações de imunodeprimidos e/ou imunossuprimidos, principalmente em portadores de HIV/AIDS. Entretanto, há poucas informações sobre pacientes renais, e inclusive, nesta RSL não se encontrou nenhum relato específico sobre crianças hemodialisadas.

Os estudos selecionados (Quadro 1) tratam de pacientes hemodialisados, sem distinção da faixa etária, e em alguns deles, não há identificação da idade da população estudada. Importante faz-se a discussão, uma vez que comprovam a existência de parasitos entéricos oportunistas em populações de imunossuprimidos, como os hemodialisados, e demonstram ainda, que as colorações específicas continuam sendo o método de identificação predominante para estes parasitos. Diante dessas considerações, por consenso entre o grupo de pesquisadores, decidiu-se sobre a seleção destes estudos, independentemente da faixa etária.

Estudo realizado no Brasil(22) mostrou a prevalência de parasitismo intestinal e sintomatologia associada em pacientes submetidos à hemodiálise. Nas amostras fecais foi utilizado para identificação microscópica de parasitos o método de concentração formol-éter e para o diagnóstico de Cryptosporidium, a técnica ELISA. Das 110 amostras fecais dos pacientes hemodialisados, 51,6\% apresentaram parasitos intestinais, $26,4 \%$ e $24,5 \%$ apresentaram Cryptosporidium e Blastocystis, respectivamente, sendo que $73,6 \%$ dos pacientes com insuficiência renal crônica eram sintomáticos e, dentre estes $54,3 \%$ apresentaram monoparasitismo ou 
poliparasitismo. Das 86 fezes coletadas do grupo do grupo controle, $61,6 \%$ apresentaram parasitas intestinais e Blastocystis foi a infecção mais comum $(41,9 \%)$, sendo que $91,9 \%$ deste grupo eram sintomáticos e $60,8 \%$ apresentaram, pelo menos, um tipo de parasito. Ao comparar a prevalência de parasitos em ambos os grupos, verificou-se a presença de Cryptosporidium apenas no grupo de hemodialisados $(p<0,0001)$ e que Blastocystis hominis e Entamoeba coli foram significativamente prevalentes no grupo controle $(p<0,05)$.

Pesquisa(13) que focalizou a infecção de parasitos oportunistas em um grupo de imunocomprometidos no Egito, não traz informações sobre a faixa etária estudada. Dentre os indivíduos estudados, encontravam-se 40 casos de pacientes com câncer, 30 casos de diabetes mellitus, 30 casos com falência renal crônica em comparação a 20 indivíduos saudáveis. Os métodos parasitológicos utilizados para a detecção dos protozoários foram exame a fresco, semeaduras coradas pelo lodo, pelo ZiehlNeelsen modificado e pela Safranina-Azul de Metileno. Dos 100 pacientes do grupo-teste, foram detectadas 10 (10\%) de amostras positivas para Giardia lamblia, sete (7,0\%) positivas para Cryptosporidium parvum, três (3,0\%) positivas para Cyclospora cayetanensis, duas (2,0\%) positivas para Microsporidium, duas (2,0\%) positivas para Cyclospora e Cryptosporidium, simultaneamente. Os resultados indicaram a ocorrência de parasitos oportunistas em $30 \%$ dos pacientes imunocomprometidos e $10 \%$ no grupo de pacientes saudáveis.

Outro estudo brasileiro ${ }^{(14)}$ realizado com pacientes de 21 a 82 anos submetidos à hemodiálise, na cidade de Campo Mourão, Paraná, Brasil, detectou, com maior frequência, a presença de Blastocystis sp, Endolimax nana, Cryptosporidium sp e Entamoeba coli. Foram analisadas amostras fecais de 86 pacientes hemodialisados em comparação a 146 indivíduos saudáveis. No grupo de hemodialisados, $33(45,1 \%)$ apresentaram tais parasitos, dos quais oito $(9,2 \%)$ pacientes apresentaram mais de uma espécie. Blastocystis hominis foi encontrado em 18 (20,9\%) pacientes; Endolimax nana foi encontrado em 14 (16,3\%); Cryptosporidium parvum em dois (4,7\%); Entamoeba coli em quatro (4,7\%). Houve diferença significativa em relação ao monoparasitismo e poliparasitismo entre o grupo de hemodialisados e o grupo de referência $(p=0,0019$ e $p=0,0318$, respectivamente).

A taxa de prevalência da infecção por Cryptosporidium em 104 pacientes hemodialisados foi objeto de um estudo realizado no Irã ${ }^{(15)}$ que comparou uma população de 91 membros familiares e outra população normal constituída por 140 indivíduos, de 20 a 60 anos. Desse total, estavam infectados por Cryptosporidium: $12(11,5 \%)$ pacientes na população de hemodialisados; quatro $(4,4 \%)$ indivíduos na população familiar; e cinco (3,6\%) indivíduos na população normal. A diferença na taxa de prevalência foi estatisticamente significativa $(p<0,05)$ na comparação entre os hemodialisados e os outros grupos pesquisados. Não ocorreu diferença significativa entre os dois grupos controles e correlações entre sexo, idade, duração da diálise, história de transplante de rins ou do tratamento com drogas imunossupressoras em todos os grupos. Concluíram que a taxa de prevalência da infecção por Cryptosporidium é consideravelmente mais alta em pacientes submetidos à hemodiálise do que na população em geral, inclusive, havendo maior taxa de infecção nos pacientes diabéticos submetidos à hemodiálise.

Para determinar a frequência de Cryptosporidium em pacientes, de 30 a 49 anos, foram coletadas 50 amostras fecais de três populações distintas: 10 indivíduos portadores de câncer, 20 diabéticos e 20 dialisados, durante os meses de julho a dezembro de 2003, no Paquistão(16). Desse total de amostras, Cryptosporidium foi encontrado em 20 (40\%) dos casos, sendo oito (80\%) em pacientes com câncer, cinco (25\%) em pacientes diabéticos e sete (35\%) em pacientes dialisados. Houve maior detecção do Cryptosporidium em pacientes do sexo masculino (87\%). Segundo os autores, dentre os pacientes, os portadores de câncer têm maior probabilidade de infecção por Cryptosporidium em virtude da maior debilidade do sistema imune.

Na Turquia(17) foi investigada a prevalência da infecção por Cryptosporidium em pacientes hemodialisados adultos, na Turkia, numa população de 74 pacientes com falência renal em estágio terminal hemodialisados crônicos, comparada a uma população de 50 indivíduos saudáveis. Dentro do grupo de 74 hemodialisados, 15 (20,27\%) apresentaram oocistos de Cryptosporidium, por sua vez, dentro do grupo de 50 indivíduos saudáveis, não ocorreu qualquer tipo de infecção por Cryptosporidium, apresentando diferença 
estatisticamente significante $(p<0,0001)$. Em 10 dos 15 hemodialisados e positivos para Cryptosporidium não havia sintomas, sendo que os cinco restantes apresentaram diarreia. Dos cinco pacientes diarréicos, quatro foram positivos para Cryptosporidium, sendo um positivo para Cryptosporidium e Giardia lamblia.

Outra investigação brasileira ${ }^{(18)}$ determinou a frequência de Cryptosporidium parvum em um grupo de 23 pacientes adultos submetidos à hemodiálise e um grupo de 32 pacientes com transplante renal comparados a uma população de 27 pacientes com hipertensão arterial sistêmica. Em uma pesquisa realizada num período de 18 meses, em Uberaba, Brasil. Foram coletadas de uma a seis amostras de cada paciente e detectadas frequências de Cryptosporidium parvum em $34,8 \%, 25 \%$ e $17,4 \%$ dos pacientes transplantados, hemodialisados e com hipertensão, respectivamente, sendo que não houve diferença significativa entre os três grupos, mas ocorreu uma maior frequência significativa no grupo de transplantados renais.

Uma pesquisa que analisou a criptosporidiose e outras parasitoses em pacientes hemodialisados ou submetidos a transplante renal no Brasil( ${ }^{(19)}$ trabalhou com três grupos de pacientes adultos que foram selecionados para a pesquisa, dentre eles, um grupo-teste constituído por 23 pacientes com insuficiência renal crônica e 32 pacientes submetidos a transplante e outro grupo constituído por 27 pacientes com hipertensão arterial sistêmica (grupo controle). Nessa pesquisa, cada paciente foi submetido a números variáveis de exames. Dos 23 pacientes transplantados, oito $(34,8 \%)$ foram positivos para Cryptosporidium parvum e dois (8,7\%) para Endolimax nana; dos 32 pacientes hemodialisados, oito (25.0\%) foram positivos para Cryptosporidium parvum e 11 (34,4\%) positivos para outros parasitos (Endolimax nana (3), Entamoeba coli (1), E. histolytica (1), Giardia lamblia (1), Ascaris lumbricoides (2), Trichuris trichiura (1), Strongyloides stercoralis (1), Schistosoma mansoni (1)); dos 27 indivíduos do grupo controle, $4(17,4 \%)$ foram positivos para Cryptosporidium parvum e $7(25,9 \%)$ positivos para outros enteroparasitos (Endolimax nana (4), Entamoeba coli (1), Giardia lamblia (1), Trichuris trichiura (1).

Em pesquisa desenvolvida no Egito(20), determinou-se o potencial patogênico dos parasitos entéricos oportunistas em 187 pacientes com câncer com ou sem uso de medicamentos imunossupressivos, 80 pacientes com falência renal crônica, 100 pacientes com diabetes mellitus e 60 pacientes submetidos à terapia por corticóides, totalizando 427 pacientes na fase adulta. Foi analisada uma amostra fecal de cada paciente, resultando na detecção de parasitos entéricos oportunistas em 98 amostras fecais, com a prevalência de $23 \%$, sendo 19 $(31,7 \%)$ em pacientes em uso de corticóides, 48 (25,7\%) em pacientes com câncer, 23 (28,7\%) em pacientes com falência renal crônica e oito $(8,0 \%)$ em pacientes diabéticos. Ocorreu o predomínio da infecção no sexo masculino $(32,6 \%)$ em relação ao sexo feminino. Foi constatada uma associação estatisticamente significativa de infecção por Giardia lamblia e Cryptosporidium. Segundo os autores, não ocorreu diferença significativa entre os três grupos examinados $(p=0,09)$ na detecção por Cryptosporidium parvum. Entretanto, em relação ao grupo de pacientes submetidos a transplante renal verificou-se frequência significativamente maior $(p<0,01)$ de amostras positivas para Cryptosporidium parvum quando comparada com outras parasitoses, que tiveram frequência inferior $(p<0,01)$.

Outro estudo também realizado no Egito(21) com pacientes de 40 a 60 anos, no Egito, para a identificação de protozoários em 120 pacientes renais crônicos submetidos à hemodiálise (75 do sexo masculino e 45 do sexo feminino) em comparação a 40 pacientes imunocompetentes com complicações de diarreia, foi coletada uma amostra fecal de cada paciente. Tais amostras foram examinadas por exame direto, concentradas em formol-éter e coradas pelos métodos de Giemsa, Ziehl-Neelsen modificado, coloração de metilvioleta e anilina carboxílica- Oocistos de Cryptosporidium sp foram identificados em 18/120 (15\%) dos pacientes hemodialisados, dentre estes, $11(9,2 \%)$ tinham somente Cryptosporidium e sete $(5,8 \%)$ apresentaram parasitos adicionais (três com Giardia lamblia, um com Entamoeba histolytica e três com Cyclospora e Microporidium). Foram também, detectados oocisto de Cryptosporidium em 2/40 $(5,0 \%)$ dos pacientes imunocompetentes. Oocistos de Cyclospora foram detectados em 9/120 (7,5\%) dos pacientes hemodialisados com quatro $(3,3 \%)$ amostras exclusivamente positivas para Cyclospora e cinco (4,2\%) amostras positivas para Cyclospora e Cryptosporidium, simultaneamente. Isospora belli foi detectada em 3/120 $(2,5 \%)$ dos casos de hemodialisados, com um $(0,83 \%)$ caso 
positivo para Isospora belli e dois $(1,67 \%)$ positivas para Isospora belli, Cryptosporidium e Microsporidium. A infecção por Cryptosporidium parvum foi detectada em oito $(34,8 \%)$ dos pacientes transplantados, oito $(25,0 \%)$ dos pacientes hemodialisados e quatro $(17,4 \%)$ dos indivíduos do grupo controle. Outras parasitoses foram detectadas em dois (8,7\%) dos transplantados, 11 (34,4\%) dos hemodialisados e sete $(25,9 \%)$ do grupo controle.

Apesar desses estudos não se referirem a crianças hemodialisadas, confirmam a detecção de parasitos entéricos oportunistas em populações imunossuprimidas, em especial, nos pacientes renais crônicos submetidos à hemodiálise. Reforçam, ainda, a importância de pesquisas sobre tais parasitos e a utilização de técnicas específicas para o diagnóstico.

\section{CONCLUSÃO}

Dentre os estudos analisados, os pesquisadores verificaram que os pacientes submetidos à hemodiálise são potencialmente infectados por agentes entéricos oportunistas. Tal susceptibilidade pode ser devida ao fato

\section{REFERÊNCIAS}

1. Salgado N, Filho; Brito DJA. Doença Renal Crônica: a grande epidemia deste milênio. J. Bras. Nefrol. 2006;28(3 Suppl 2):1-5. 2. Marques $A B$, Pereira $D C$, Ribeiro RC. Motivos e frequência de internação dos pacientes com IRC em tratamento hemodialítico. Arq. Ciênc. Saúde 2005;12(2):67-72.

3. National Kidney Foundation. K/DOQI Clinical Practice Guidelines for Chronic Kidney Disease: Executive Summary New York; 2002.

4. Ludwig, KM, Alvares F, Filho; Ribeiro-Paes JT. Correlação entre condições de saneamento básico e parasitoses intestinais na população de Assis, Estado de São Paulo. Rev. Soc. Bras. Med. Trop. 1999;32(5):547-55.

5. Ferreira CB, Marçal O, Jr. Enteroparasitoses em escolares do Distrito de Martinésia, Uberlândia, MG: um estudo piloto. Rev.

Soc. Bras. Med. Trop. 1997;30(5):373-7.

6. Carneiro JR, Rodrigues N; Lima JD; Evangelista PL. Prevalência da Criptosporidiose em crianças procedentes da região metropolitana de Goiânia-GO. Rev. Pat. Trop. 1995;24(2):243-53.

7. García-Zapata, M. T., Manzi, R. S. Souza E. S, Júnior. Parasitosis emergentes y reemergentes de interes sanitário en el sistema único de salud (SUS) en el Estado de Goiás, Brasil: Importancia de su diagnóstico y seguimient. Sociedad Iberoamericana de Información Científica, Buenos Aires, Argentina, 2002. Avaible from: http://www. siisalud.com.

8. Hamour AA \& Mandal BK. Coccidian parasites in patients with AIDS: Cryptosporidiosis, Microsporidiosis, Isosporiasis and Cyclosporiasis. Balliere's Clinical Infectious Diseases. 1996;3(1):137-53.

9. Souza RQ, Torres LM, Graziano KU, Turrini RNT. Microorganismos da subclasse coccidia:resistência e implicações para o processamento de materiais de assistência à saúde. Rev Esc Enferm USP 2012; 46(2):466-71. desses indivíduos serem submetidos a terapias imunossupressivas.

Considerando o aumento de doenças como as nefropatias crônicas em escala mundial são necessários estudos mais aprofundados sobre as infecções oportunistas que acometem tal população, a fim de se enfrentar diretamente os desafios da saúde pública de ordem econômica e social, com a diminuição de custos relacionados aos tratamentos e a melhora da qualidade de vida dos pacientes.

Essa revisão mostrou ainda que há necessidade de que a população de hemodialisados seja considerada como grupo de risco para as infecções oportunistas, sintomáticas ou assintomáticas, causadas por Cryptosporidium sp, Cyclospora cayetanensis, Isospora belli e Blastocystis hominis.

Assim, é preciso ser recomendada a realização periódica de exames coprológicos para a detecção de protozoários e tratamento da população em estudo, a fim de se evitar também a contaminação de ambientes hospitalares.

10. Munoz SI, Takayanagui AM, Santos CB, Sanchez-Sweatman O. Revisão sistemática de literatura e metanálise: noções básicas sobre seu desenho, interpretação e aplicação na área da saúde. In: Brazilian nursing Communication Symposium. São Paulo, 2002.

11. Sampaio, R. F; Mancini, M. C. Estudos de revisão sistemática: um guia para síntese criteriosa da evidência científica / Systematic review studies: a guide for careful synthesis of the scientific evidence. Rev. Bras. Fisioter. 2007;11(1):83-9.

12. Polit, DF; Beck CT; Hungler, BP. Fundamentos de pesquisa em enfermagem: métodos, avaliação e utilização. 5ed. Porto Alegre: Artmed, 2004.

13. Baiomy AM; Mohamed KA; Ghannam MA; Shahat SA; AlSaadawy AS. Opportunistic parasitic infections among immunocompromised Egyptian patients. J Egypt Soc Parasitol. 2010;40(3):797-808.

14. Kulik RA; Falavigna DL; Nishi L; Araujo SM. Blastocystis sp. and other intestinal parasites in hemodialysis patients. Bras $\mathrm{J}$ Infectar Dis. 2008;12(4):338-41.

15. Seyrafian S; Pestehchian N; Kerdegari M; Yousefi HA; Bastani B. Prevalence rate of Cryptosporidium infection in hemodialysis patients in Iran. Hemodial Int, 2006;10:375-9.

16. Baqai R; Anwar S; Kazmi SU. Detection of cryptosporidium in immunosuppressed patients. J Ayub Meb Coll Abbottabad. 2005;17(3):38-40.

17. Turkcapar N; Kutlay S; Nergizoglu G; Atli T; Duman N. Prevalence of Cryptosporidium infection in hemodialysis patients. Nephron. 2002;90(3):344-6.

18. Chieffi PP; Sens YA; Paschoalotti MA; Miorin LA; Silva HG; Jabur $\mathrm{P}$. Infection by Cryptosporidium parvum in renal patients submitted to renal transplant or hemodialysis. Rev. Soc. Bras. Med. Tropjul. 1998;31(4):333-7.

19. Jabur P, Miorin, LA; Silva HG, Paschoalotti MA; Chieffi PP, Sens YA. Criptosporidiose e outras enteroparasitoses em 
pacientes submetidos a transplante renal ou hemodiálise/

Cryptosporidiosis and others intestinal parasitic deseases in patients submited to kidney transplantation or hemodialysis. J.

Bras. Nefrol. 1996;18(3):239-242.

20. Abaza SM; Makhlouf LM; El-Shewy KA; El-Moamly AA.

Intestinal opportunistic parasites among different groups of immunocompromised hosts. J Egypt Soc Parasitol.

1995;25(3):713-27.

21. Ali MS; Mahmoud LA; Abaza BE; Ramadan MA. Intestinal spore-forming protozoa among patients suffering from chronic renal failure. J Egypt Soc Parasitol. 2000;30(1):93-100.

22. Gil FF; Barros MJ; Macedo NA; Júnior CGE; Redoan R; Busatti $\mathrm{H}$; et al. Prevalence of intestinal parasitism and associated symptomatology among hemodialysis patients. Rev.Inst. Med. Trop. 2013;55(2):69-74.

Artigo recebido em 12/11/2012.

Aprovado para publicação em 11/06/2013.

Artigo publicado em 31/12/2013. 